\title{
DESIGN OF A QUADRAMANUAL ROBOT FOR SINGLE-NOSTRIL SKULL BASE SURGERY
}

\author{
Philip J. Swaney* \\ Jordan M. Croom \\ Jessica Burgner \\ Hunter B. Gilbert \\ D. Caleb Rucker \\ Robert J. Webster III \\ Department of Mechanical Engineering \\ Vanderbilt University \\ Nashville, Tennessee, 37212 \\ philip.j.swaney@vanderbilt.edu
}

\author{
Kyle D. Weaver \\ Department of Neurological Surgery \\ Vanderbilt University Medical Center \\ Nashville, Tennessee, 37232
}

\author{
Paul T. Russell III \\ Department of Otolaryngology \\ Vanderbilt University Medical Center \\ Nashville, Tennessee, 37232
}

\begin{abstract}
Innovations in the field of continuum robotics are enabling increasingly thin and dexterous slave robots in surgical telemanipulation systems. Actuation of these robots is a challenge, because many degrees of freedom must be controlled simultaneously in a compact and unobtrusive package in the operating room. While significant progress in designing these actuation units has been made for both larger, non-medical continuum robots, and for certain types of surgical continuum robots, the design of clinically-relevant actuation units for concentric tube robots is a comparatively understudied area of research. To address this, in this paper we present the design of a robot intended to deliver four concentric tube manipulators simultaneously through a single nostril. We present the considerations that motivated the design and the prototype we have constructed to meet these needs.
\end{abstract}

\section{INTRODUCTION}

Design of surgical robot slave manipulators has recently been moving toward more compact devices [1,2], and also away from general purpose robots in favor of systems designed and built with one particular surgical access route or type of surgical procedure in mind (see e.g., [3-5]). There has also been a trend toward using continuously flexible robots (i.e., "continuum robots") to enhance dexterity inside the patient in small-diameter

\footnotetext{
*Address all correspondence to this author.
}

packages (see e.g., [6-8]). For a recent review of a variety of surgical robotic designs and applications see [9].

Due to the infinite degree of freedom (DOF) configuration space and the high degree of freedom actuation space of continuum robots (see [10] for a review), designing actuation systems for these robots is challenging. Many novel continuum and hyperredundant robot designs can be found in [11]. One way redundant robots can be actuated is with motors embedded within the arm itself [12]. A design challenge with this 12 DOF robot was to locate motors compactly within the arm, which was accomplished by designing novel joints. Another approach is to locate all actuators at the base of the arm. This was done in the hyperredundant Clemson Elephant's Trunk robot [13], which was actuated by tendons that run along its length and apply forces and moments to a 32 DOF backbone constructed from universal joints. The tendons are actuated by DC motors and design challenges included motor placement, cable routing, and the need for a tensioning mechanism to remove cable slack. A similar style of robot foregoes a backbone in favor of centrally located, pressurized chambers, while retaining tendons $[14,15]$. The scalability of tendon actuation has been demonstrated in a $4 \mathrm{~mm}$ diameter tendon-actuated continuously flexible (i.e., infinite DOF) continuum robot catheter for surgical applications [16].

The requirements of the surgical environment (sterility, ease of use, safety, compactness, etc.) place stringent design requirements on continuum robot actuation systems. Another example of a medical continuum robot designed with these considerations 


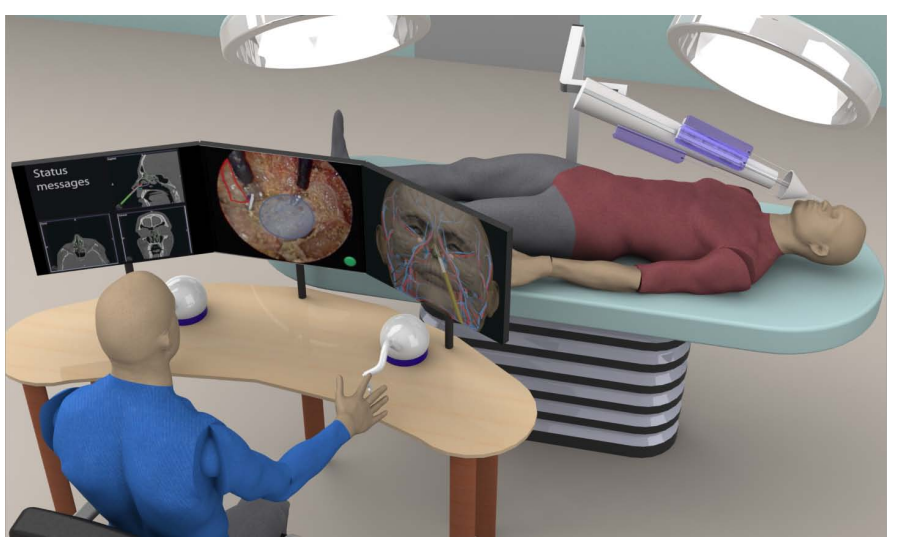

Figure 1. Conceptual drawing of the quadramanual system with a surgeon's console showing the tools in the skull base and the cannula paths through the nasal cavity.

in mind uses multiple backbones which can be actuated in both tension and compression. A compact actuation unit based on miniature DC motors and lead screws was designed to actuate the backbones [6]. This robot was designed for bimanual throat surgery, and a similar device has been constructed for bimanual single port abdominal surgery [17].

In this paper we consider a smaller natural orifice, the nostril. A robot designed to work with multiple manipulators through this constrained entry point could have significant clinical impact because of the high incidence of disease in this area of the body, and the challenge of using traditional instruments by hand here. Among the many procedures that could benefit from an endonasal robot is pituitary tumor resection; these tumors account for $15-20 \%$ of all primary brain tumors [18]. Endonasal skull base surgery is currently emerging from conventional transfacial and transcranial approaches as a much less invasive (but more technically challenging for the physician) alternative [19].

The surgery has many challenges due to the confined spaces involved, and the lack of dexterity (see $[20,21]$ ) of current rigid surgical tools, which lack wrists or other means of controllable deflection. One challenge is in the initial drilling away of bone to enlarge the nasal passage (when required) and to expose the surgical site. A robotic system has been designed to assist with this initial phase of the surgery, assisting the surgeon in guiding the drill [22]. A surgical system designed to be deployed after the surgical site is exposed has recently been proposed by our group, to assist with tumor resection and other surgical objectives [23] (see Fig. 1). The robot's manipulators consist of needle-diameter concentric tube continuum robots [24,25] (see Fig. 2), which have dexterity analogous to tentacles. The concentric tube design enables manipulators to be sufficiently small and dexterous that multiple instruments can be inserted and manipulated through a single nostril.

Contribution: Mechanical design of the actuation unit itself was not a focus of the system-level presentation in [23], where a

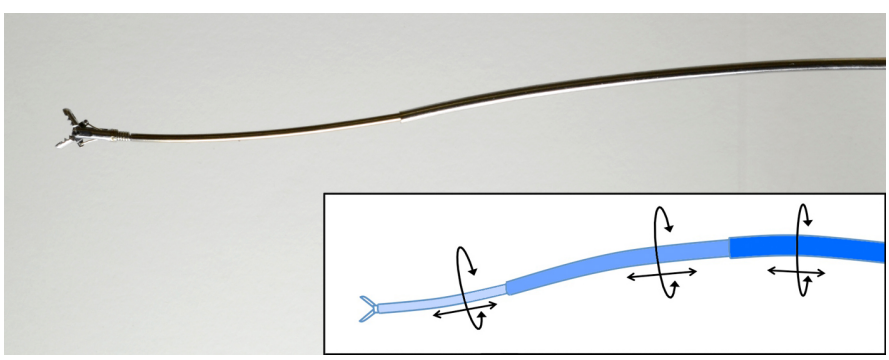

Figure 2. Photograph of a needle-diameter concentric tube robot with grasping forceps attached. The inset figure shows the 6 DOF of this robot arm. Our system will introduce four of these robot arms through a single nostril for skull base surgery.

preliminary prototype was used simply to illustrate proof-ofconcept, and mechanical design considerations for the actuation unit were beyond the scope of the paper. Herein, we focus specifically on those aspects of the system. Our contributions include (1) a discussion of the design considerations for the slave robot, and (2) a description of a novel robotic actuation unit designed to address them. The most important differences of the new robot compared to the preliminary prototype of [23] is that the new robot has four arms (rather than two), and the arms are spaced closer to one another, enabling them all to be inserted through a single nostril, making the new design an important step toward clinical application.

\section{SYSTEM CONCEPT}

Endonasal skull base surgery requires tools that can work dexterously at the skull base, reaching around protrusions of bone, to access tumors and other structures. We envision a robotic system conceptually similar to the da Vinci robot [26], in that the surgeon is seated at a master console, controlling a remote slave that manipulates instruments that enter the patient and perform surgery under physician control (see Fig. 1).

Such a system requires a camera for visual feedback and one or more surgical instruments, as well as suction/irrigation. We chose to include a camera and three instruments in our system, to enable simultaneous use of two surgical tools with suction/irrigation and a camera for visual guidance. All of these tools are mounted at the tip of concentric tube manipulators, including the camera, enabling the surgeon to control view angles and visualize areas that may be occluded from the view of a traditional straight endoscope.

\section{DESIGN SPECIFICATIONS AND CONSIDERATIONS}

To design a robot for endonasal skull base surgery, we identified the following design specifications and requirements:

Required Velocities: In order to obtain an estimate of the velocities used in skull base surgery we asked an experienced surgeon to move a Phantom Omni haptic device (Sensable, Inc. USA) to mimic the peak instrument velocity used in skull base 
(a)

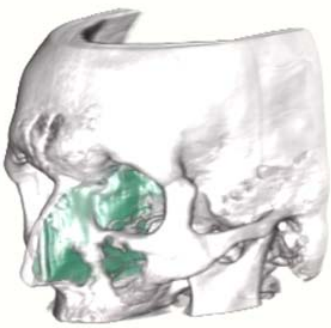

(b)

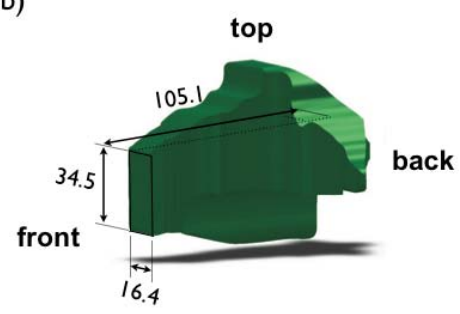

Figure 3. (a) Workspace (green) through one nostril for endonasal skull base surgery on an average-sized human. (b) Working volume dimensions (in $\mathrm{mm}$ ). This orientation is the same as that of (a).

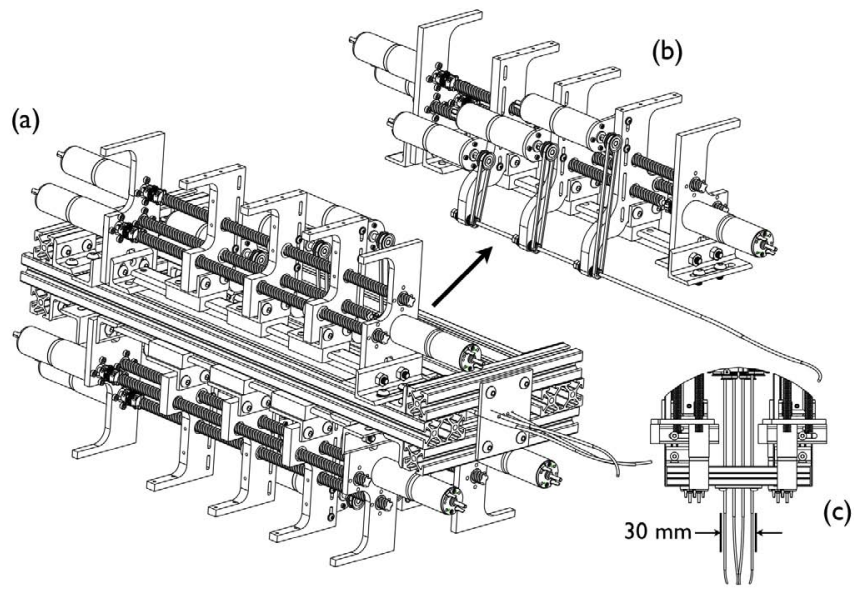

Figure 4. (a) Quadramanual robot with (b) one arm disassembled also showing (c) an overhead view of all tubes exiting the robot within the anatomical nostril constraint.

surgery. The peak velocity was measured to be $1.8 \mathrm{~cm} / \mathrm{sec}$. While further investigation is needed to completely characterize necessary velocities, this provides a rough estimate for design purposes. We will design our actuation unit to be capable of many times this peak velocity to account for the uncertainty in how this measurement maps to required tool tip velocities in surgery.

Anatomical Constraints: As reported in [23] segmented medical images from one example computed tomography scan reveal the workspace dimensions shown in Fig. 3 for singlenostril endonasal access to a pituitary tumor. The most constrained area of this workspace is the nostril opening which is approximately $16 \mathrm{~mm}$ by $35 \mathrm{~mm}$. We note that there is some anatomical variation and that in the future we intend to segment multiple image sets to characterize it. This initial image was selected by experienced physicians as an approximately average anatomical example. All surgical instruments must enter through the nostril opening, and they must be sufficiently long to access the rear wall of the cavity and remove tumor tissue there. The actuation unit prototype described in subsequent sections is designed such that travel lengths of each concentric tube manipulator can be adjusted without changing the overall design concept.

Required Forces: Although the forces applied during manual skull base surgery have yet to be quantitatively measured, we estimated them based on clinical experience. We asked a trained physician to push on a force sensor with a simulated surgical instrument using the maximum force he would normally use in surgical resection of a pituitary tumor. This resulted in a force of $0.7 \mathrm{~N}$. While further investigation is needed to more completely characterize necessary forces, including outfitting endonasal surgical tools with force sensors, this provides a rough estimate for our design. As described in subsequent sections, we will design our actuation unit to be capable of many times this level of force application, to account for the uncertainty in how this limited measurement maps to actual force application during surgery.

Surgical Workflow: As mentioned in the introduction, the robot is designed to be deployed after the pituitary tumor has been exposed by initial drilling and bone/cartilage removal procedures done in standard endonasal surgery. Our actuation unit will be mounted to a support arm that is capable of positioning it over the patient. The arm will be locked into place, and the concentric tube manipulators deployed.

Degrees of Freedom: Although some studies exist on optimization of concentric tube robots given task constraints $[23,27$, 28], significant future research is needed in this area. For example, it remains to be demonstrated that it is possible in physical hardware to construct multiple dominant stiffness tube pairs (as some prior methods require) and to be determined how one may include angular considerations in objective functions for manipulators (some prior studies include only trajectory or positionbased objectives). Thus, in our prototype, we choose three tubes ( 6 degrees of actuation freedom) per concentric tube manipulator. This number is selected somewhat arbitrarily, based on our experience that three tube manipulators appear to be a reasonable tradeoff between dexterity and actuation complexity. However, we note that the basic design of our actuation unit described in subsequent sections is amenable to adding additional tubes in the future, if required, without changing the basic design.

\section{ROBOT DESIGN}

Based on these design considerations, a robot capable of delivering up to four surgical instruments to the skull base through a single nostril was designed. Each surgical instrument is delivered to the site via a manipulator module, with each six degree of freedom module controlling a single concentric tube manipulator. The entire robot consists of four copies of this manipulator module that are mirrored with respect to one another (see CAD drawing in Fig. 4), allowing the tube bases to exit the robot within the $16 \mathrm{~mm}$ by $35 \mathrm{~mm}$ opening.

The four manipulator modules are supported by a frame constructed from extruded aluminum (80/20 Inc., USA, shown in Fig. 5). This frame serves the secondary purpose of providing a convenient method for mounting the robot to a support arm in the operating room. Since the individual extruded members use slotted connections, the clearances and alignment between ma- 


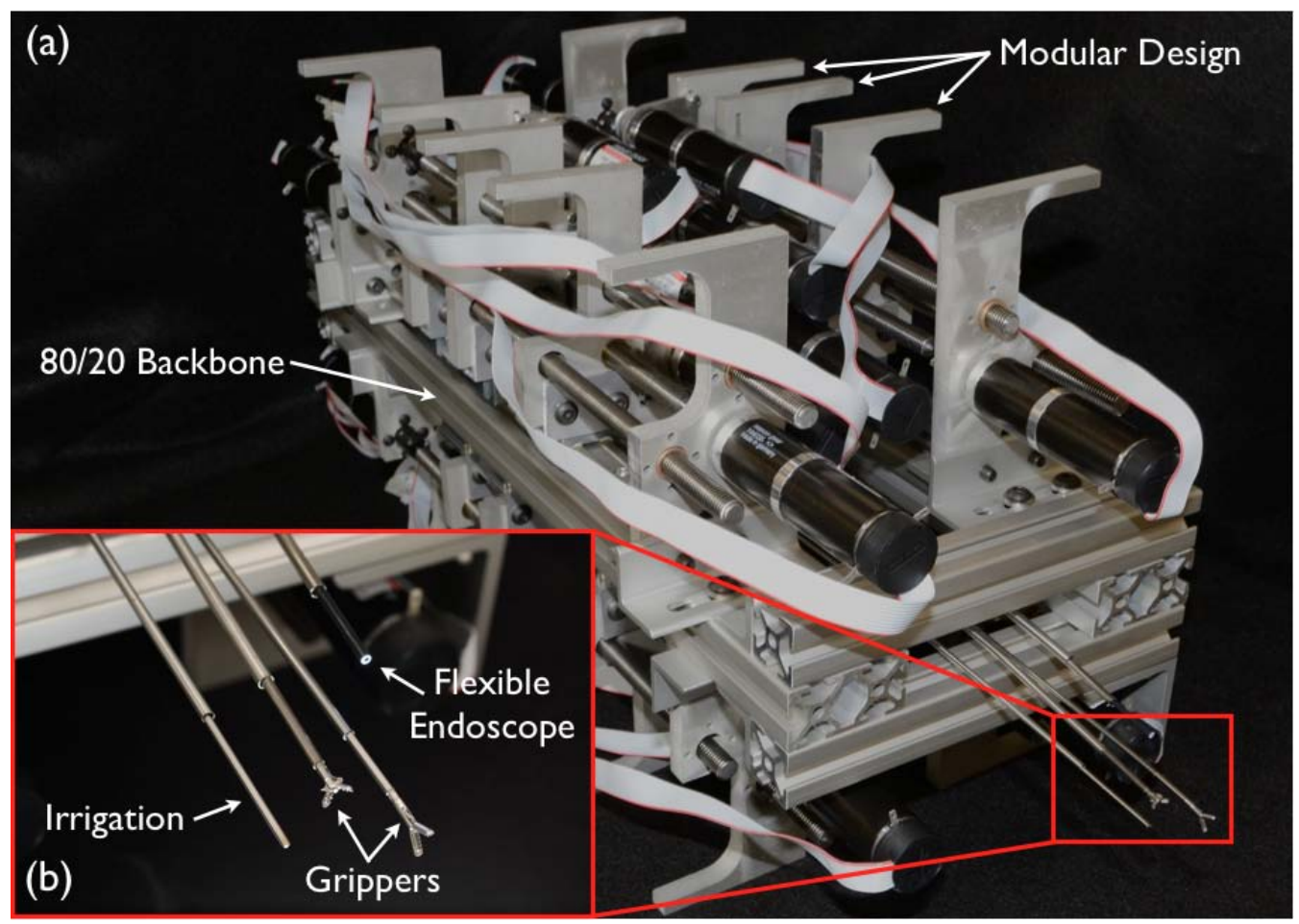

Figure 5. (a) Photograph of the complete quadramanual robot (b) Close-up view of the active cannula end effectors (2 grippers, suction, and flexible endoscope with light source). These are shown in a straight configuration, but can be precurved to enable tentacle-like motion.

nipulator modules can be easily adjusted. The modular nature of the design also allows for each manipulator module to be removed/replaced without having to disassemble the entire robot.

\section{Manipulator Module Design}

Each of the four manipulator modules contains three identical single-tube stages (Fig. 6). Each single-tube stage controls a single tube in two degrees of freedom, namely axial translation and axial rotation. The single-tube stage translates via a captive acme nut that rides on a lead screw actuated remotely by a DC motor (motor selection is addressed in Section ). Rotation is accomplished by a motor-pulley-belt system mounted to the stage. Each stage is mounted to a PTFE-lined sleeve-bearing, which rides on a shared track (i.e., all three single-tube stages in a given manipulator module share the same track).

Each single-tube stage is designed to accommodate a range of tube sizes, since future studies will be needed to determine optimal tube sizes, curvatures, and lengths for skull base surgery. In previous concentric tube robot actuation units, adaptation to different size tubes was accomplished by a collet system, where if a new tube diameter was needed one needed only to replace one commercially available collet with another collet size [23]. While this tube gripping mechanism design is highly flexible, it requires the manipulator modules to hold their respective tubes at a distance of at least one collet diameter from one another. Since we wish to minimize this intra-manipulator distance, instead of

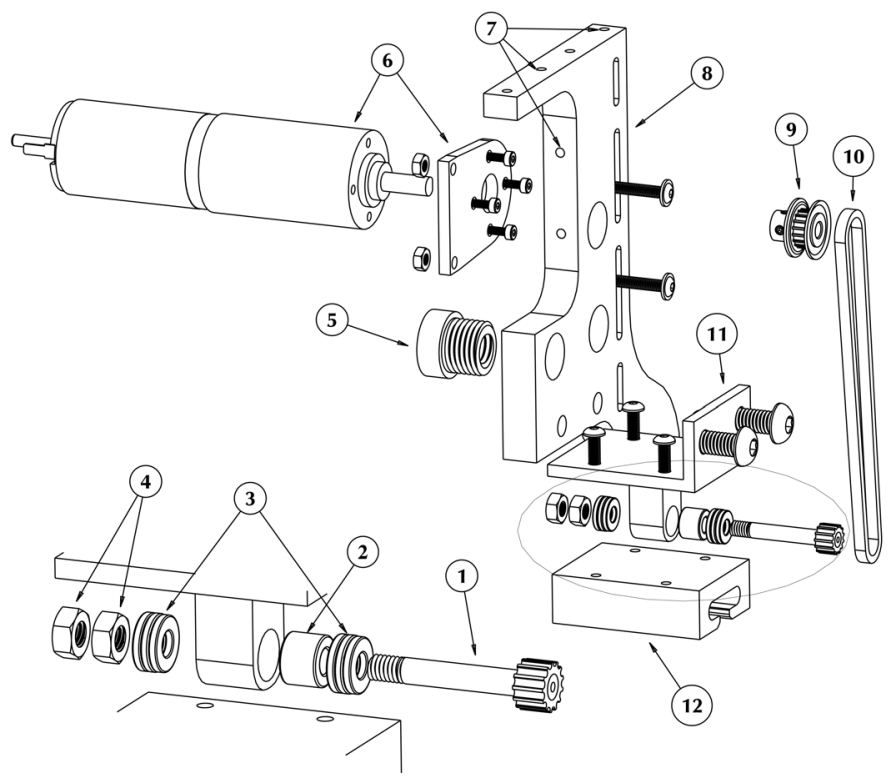

Figure 6. Single-tube stage assembly. Exploded view with (1) cannula holder assembly, (2) sleeve bearing, (3) thrust bearings, (4) jam nuts, (5) acme nut, (6) rotation motor and bracket, (7) cable carrier mounting holes, (8) cannula stage body, (9) motor timing pulley, (10) timing belt, (11) stage mounting bracket, and (12) carriage. 
collets, each tube was permanently bonded to a cylindrical brass housing ( $4 \mathrm{~mm} \mathrm{OD}$ ). Each brass housing was bonded to an aluminum pulley on one face, and threaded on the other to secure it to the single-tube stage (Fig. 6). In the assembled device, the brass housing around the tube is supported inside a low-friction sleeve bearing, and secured axially against front and rear thrust bearings by two M4 jam nuts.

\section{Motor Placement, Linear Translation, and Limit Switches}

Up to three single-tube stages can be mounted on a single manipulator module in our current embodiment (Fig. 7). Staggering the motors on each single-tube stage in the three possible mounting locations in the lateral direction (with respect to the tube axis), implies that the minimum distance between stages is limited only by the thickness of the linear bearings that support the single-tube stage on the track. Each stage contains integrated collision-detection limit switches, which trigger soft stops on the robot controller. The front end plate on the single track assembly supports the three lead screws via bronze bushings. The lead screw motors (which accomplish translation of each single-tube stage) are mounted to the front and rear end plates. Each motor is coupled to a lead screw using a flexible coupling which supports axial and radial loads exerted on the screw. Cable carriers can also be attached to each single-tube stage, as well as the rear end plate, for cable management. A slotted bracket affixes the track to the support frame, enabling horizontal adjustments of \pm $10 \mathrm{~mm}$.

\section{Some Remarks on Tubes}

As mentioned previously, tube design given anatomical constraints and surgical objectives is an open research topic, though some preliminary work has been done in this area [23, 27, 28]. The tubes used have thus far been made of nitinol, but it has been noted that other materials that can sustain high strain without elastic deformation are also applicable. Design parameters available are tube diameters and wall thicknesses (which affect the overall stiffness of the device), tube curvatures (each tube may be preshaped with a general space curve by a heat treatment process), tube lengths, and number of tubes. The maximum curvature possible in each tube is a function of the maximum recoverable strain and tube diameter [29]. The quadramanual robot is designed to accommodate a large range of possible tube designs. Different tube lengths and curved section lengths will require different amounts of travel of each single-tube stage. These travel distances can easily be adjusted by replacing the lead screws in our design with longer or shorter lead screws, with nearly all other parts remaining the same. The lead screw lengths selected in this prototype were based on the preliminary workspace design optimization in [23], plus approximately $10 \mathrm{~cm}$ of additional travel to accommodate the possibility that future tube designs may call for greater axial displacement or additional concentric tubes.

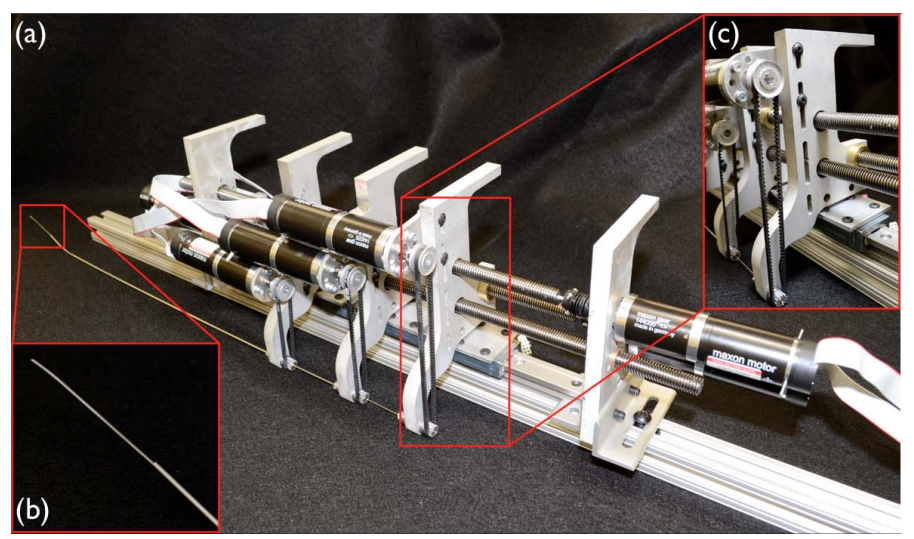

Figure 7. a) Photograph of a single manipulator module, b) a close up view of an active cannula tip and c) a single tube stage. Note: cable carriers used to organize and avoid entanglement of the wires are not shown.

\section{Actuators, Forces, and Velocities}

To actuate all 24 degrees of freedom on the quadramanual robot we selected Maxon RE 25 (\#339152, Maxon USA) motors. These motors were selected because they provide an excellent power/volume ratio. They also will be capable, over a very wide range of possible tube designs, of dramatically exceeding the specifications discussed in Section . Each motor is equipped with a 53:1 planetary gear head (\#144035, Maxon USA) and a 2000 count per revolution encoder (\#225778, Maxon USA). The translational motors are coupled to $3 / 8-8$, 4-start acme lead screws. The rotational motion of the cannula is accomplished with timing pulleys mounted to the motor and the cannula holder, with an effective gear ratio of $689: 17(\approx 40.53: 1)$.

While the mappings between actuator forces and velocities and end effector forces and velocities are complex (see [30]), we will now consider some special cases, to argue that the actuation unit itself will not be a limiting factor in achieving the requirements for any reasonable tube design. Consider a straight tube actuated by any single-tube stage of our robot. If one wished to push linearly forward with the tip of tube, at 25 times the maximum speed of $1.82 \mathrm{~cm} / \mathrm{sec}$ determined in Section, one would be able to push with a force of $15.3 \mathrm{~N}$, which is 21 times the estimated maximum force in Section. This calculation is based on the fact that the motors are rated for $20 \mathrm{~W}$, and the efficiency of the planetary gearhead is $59 \%$ and the efficiency of the lead screw was estimated to be $42 \%$ based on information in [31]. Similarly, with respect to the rotational degrees of freedom, one can consider a curved tube whose tip is deflected from the axis of the tube base. Given a desired tip linear velocity created by tube rotation of 25 times the $1.82 \mathrm{~cm} / \mathrm{sec}$ determined in Section , the rotary actuator would be capable of applying $24.8 \mathrm{~N}$ at the tip, assuming the tube were perfectly rigid, which is 34 times the estimated maximum force in Section. This calculation is once again based on the $20 \mathrm{~W}$ rated value for the motors and includes the $98 \%$ efficiency of the belt drive and 59\% efficiency of the 
planetary gearhead on the rotational degrees of freedom. Thus, there is a significant amount of power available at the tube bases, even considering the mechanism efficiencies involved.

\section{CONCLUSION AND FUTURE WORK}

In this paper we described the design of a novel 24 degree of freedom quadramanual slave robot intended to be teleoperated for use in single-nostril endonasal skull base surgery. The robot described in this paper is one component of a larger system that will include a surgeon control console conceptually similar to that of the da Vinci robot. In terms of slave robot development, in this paper we have sought a balance between compactness and adaptability to multiple future tube design sets and surgical objectives, selecting motors powerful enough to exceed our design specifications by a factor of 20 or more under typical conditions. We believe the current design is sufficiently compact for integration in the operating room, although sterility issues will need to be addressed in future work. We note that if additional compactness is a strong priority in future concentric tube robot applications, one could collect more detailed surgical velocity and force data, and from it determine the absolute minimum power/size motors capable of achieving the specifications. The next steps with the prototype robot described herein are to conduct cadaver studies to evaluate the efficacy of the robot in reaching specific anatomical locations of interest in skull base surgery and in performing surgical tasks.

Although the design presented in this paper was developed with endonasal surgery in mind, we note that with a few modifications, it could be adapted to a number of other minimallyinvasive surgical procedures. Examples include procedures in the middle ear, throat, and other neural spaces, in addition to the previously suggested applications for concentric tube robots in fetal surgery, lung surgery, and cardiac surgery, among others. Given the small envelope within which the tools exit the robot, multiple tools can be inserted through natural orifices or small incisions. Thus, our quadramanual robot design may reduce trauma, provide surgeons with the ability to navigate around sensitive structures, and improve patient recovery times through minimally invasive surgical procedures.

In endonasal surgery, the use of concentric tube robots rather than rigid surgical tools appears likely to enable physicians to reach previously inaccessible regions while minimizing damage to healthy tissues. If our system is successful in achieving this, we anticipate that it will increase the number of patients who are candidates for the natural orifice endonasal procedure, replacing the highly traumatic transcranial or transfacial approaches for these patients.

\section{ACKNOWLEDGMENT}

The authors thank Scott T. Nill for assistance in fabricating and assembling the robot. This work was supported in part by National Institutes of Health grant R21 EB011628, and in part by the National Science Foundation grants 0651803 and award IIS-1054331.

\section{REFERENCES}

[1] Parmar, A., Grant, D. G., and Loizou, P., 2010. "Robotic Surgery in Ear Nose and Throat". European Archives of Oto-Rhino-Laryngology, 267(4), pp. 625-633.

[2] Hagn, U., Konietschke, R., Tobergte, A., Nickl, M., Jörg, S., Kübler, B., Passig, G., Gröger, M., Fröhlich, F., Seibold, U., Le-Tien, L., Albu-Schäffer, A., Nothhelfer, A., Hacker, F., Grebenstein, M., and Hirzinger, G., 2010. "DLR MiroSurge: A Versatile System for Research in Endoscopic Telesurgery". International Journal of Computer Assisted Radiology and Surgery, 5(2), pp. 183-193.

[3] Miroir, M., Szewczyk, J., Mazalaigue, S., Ferrary, E., Sterkers, O., and Grayeli, A. B., 2010. "RobOtol: From Design to Evaluation of a Robot for Middle Ear Surgery". IEEE/RSJ International Conference on Intelligent Robots and Systems, pp. 850-856.

[4] Degani, A., Choset, H., Wolf, A., Ota, T., and Zenati, M. A., 2006. "Percutaneous Intrapericardial Interventions Using a Highly Articulated Robotic Probe". IEEE / RASEMBS International Conference on Biomedical Robotics and Biomechatronics, pp. 7-12.

[5] Taylor, R. H., Mittelstadt, B. D., Paul, H. A., Hanson, W., Kazanzides, P., Zuhars, J. F., Williamson, B., Musits, B. L., Glassman, E., and Bargar, W. L., 1994. "An Image-Directed Robotic System for Precise Orthopaedic Surgery". IEEE Transaction on Robotics and Automation, 10(3), pp. 261-275.

[6] Simaan, N., Xu, K., Wei, W., Kapoor, A., Kazanzides, P., Taylor, R., and Flint, P., 2009. "Design and Integration of a Telerobotic System for Minimally Invasive Surgery of the Throat". The International Journal of Robotics Research, 28(9), pp. 1134-1153.

[7] Dario, P., Paggetti, C., Troisfontaine, N., Papa, E., Ciucci, T., Carrozza, M. C., and Marcacci, M., 1997. "A Miniature Steerable End-Effector for Application in an Integrated System for Computer-Assisted Arthroscopy". IEEE International Conference on Robotics and Automation, pp. 1573-1579.

[8] Peirs, J., Reynaerts, D., Van Brussel, H., and De Gersem, G., 2003. "Design of an Advanced Tool Guiding System for Robotic Surgery". IEEE International Conference on Robotics and Automation, pp. 2651-2656.

[9] Dogangil, G., Davies, B. L., and Rodriguez y Baena, F., 2010. "A Review of Medical Robotics for Minimally Invasive Soft Tissue Surgery”. Proceedings of the Institution of Mechanical Engineers, Part H: Journal of Engineering in Medicine, 224(5), pp. 653-679.

[10] Webster III, R. J., and Jones, B. A., 2010. "Design and Kinematic Modeling of Constant Curvature Continuum Robots: A Review". The International Journal of Robotics 
Research, 29(12), pp. 1661-1683.

[11] Hirose, S., 1993. Biologically Inspired Robots: Snake-Like Locomotors and Manipulators. Oxford University Press.

[12] Shammas, E., Wolf, A., and Choset, H., 2005. "Three degrees-of-freedom joint for spatial hyper-redundant robots". Journal of Mechanism and Machine Theory, pp. $170-190$.

[13] Hannan, M., and Walker, I., 2001. "The 'Elephant Trunk' Manipulator, Design and Implementation”. IEEE/ASME International Conference on Advanced Intelligent Mechatronics, 1, pp. 14-19.

[14] Neppalli, S., and Jones, B. A., 2007. "Design, Construction, and Analysis of a Continuum Robot". IEEE/RSJ International Conference on Intelligent Robots and Systems, pp. 1503-1507.

[15] McMahan, W., Jones, B. A., and Walker, I. D., 2005. "Design and Implementation of a Multi-Section Continuum Robot: Air-Octor". IEEE/RSJ International Conference on Intelligent Robots and Systems, pp. 3345-3352.

[16] Camarillo, D., Carlson, C., and Salisbury, J., 2009. "Configuration tracking for continuum manipulators with coupled tendon drive". IEEE Transactions on Robotics, 25(4), pp. $798-808$.

[17] Ding, J., Xu, K., Goldman, R. E., Allen, P. K., Fowler, D. L., and Simaan, N., 2010. "Design, Simulation and Evaluation of Kinematic Alternatives for Insertable Robotic Effectors Platforms in Single Port Access Surgery". IEEE International Conference on Robotics and Automation, pp. 1053-1058.

[18] ABTA, 2011. "American Brain Tumor Association". from http://abta.org.

[19] Nogueira, J. F., Stamm, A., and Vellutini, E., 2010. "Evolution of Endoscopic Skull Base Surgery, Current Concepts, and Future Perspectives". Otolaryngologic Clinics of North America, 43(3), pp. 639-652.

[20] Snyderman, C. H., Pant, H., Carrau, R. L., Prevedello, D., Gardner, P., and Kassam, A. B., 2009. "What are the Limits of Endoscopic Sinus Surgery?: The Expanded Endonasal Approach to the Skull Base". The Keio Journal of Medicine, 58(3), pp. 152-160.

[21] Cavallo, L. M., Esposito, F., and Cappabianca, P., 2010. "Surgical limits in transnasal approach to opticocarotid region and planum sphenoidale: an anatomic cadaveric study". World Neurosurgery, 73(4), pp. 301-303.

[22] Matinfar, M., Baird, C., Batouli, A., Clatterbuck, R., and Kazanzides, P., 2007. "Robot-Assisted Skull Base Surgery". IEEE/RSJ International Conference on Intelligent Robots and Systems, pp. 865-870.

[23] Burgner, J., Swaney, P., Rucker, D. C., Gilbert, H. B., Nill, S. T., Russell III, P. T., Weaver, K. D., and Webster III, R. J., 2011. "A Bimanual Teleoperated System for Endonasal Skull Base Surgery". IEEE/RSJ International Conference on Intelligent Robots and Systems, pp. 2517-2523.

[24] Rucker, D. C., Jones, B. A., and Webster III, R. J., 2010.
"A Geometrically Exact Model for Externally Loaded Concentric-Tube Continuum Robots". IEEE Transactions on Robotics, 26(5), pp. 769-780.

[25] Dupont, P. E., Lock, J., Itkowitz, B., and Butler, E., 2010. "Design and Control of Concentric-Tube Robots". IEEE Transaction on Robotics, 26(2), pp. 209-225.

[26] Guthart, G., and Salisbury, J., 2000. "The Intuitive Telesurgery System: Overview and Application". IEEE International Conference on Robotics and Automation, pp. 618-621.

[27] Bedell, C., Lock, J., Gosline, A., and Dupont, P. E., 2011. "Design Optimization of Concentric Tube Robots Based on Task and Anatomical Constraints". IEEE International Conference on Robotics and Automation, pp. 398-403.

[28] Anor, T., Madsen, J. R., and Dupont, P. E., 2011. "Algorithms for Design of Continuum Robots Using the Concentric Tubes Approach: A Neurosurgical Example". IEEE International Conference on Robotics and Automation, pp. 667-673.

[29] Webster III, R. J., Romano, J. M., and Cowan, N. J., 2009. "Mechanics of Precurved-Tube Continuum Robots". IEEE Transactions on Robotics, 25(1), pp. 67-78.

[30] Rucker, D. C., and Webster III, R. J., 2011. "DeflectionBased Force Sensing for Continuum Robots: A Probabilistic Approach". pp. 3764-3769.

[31] Precision Industrial Components, 2011. "Lead Screws and Nuts: Technical Section". from http://www.picdesign.com/Images/pdfcat/section_3.pdf. 